\title{
The Bioanalysis of Targeted Nanoparticles in Monkey Plasma via LC-MS/MS
}

${ }_{4}$ Wei Song ${ }^{1}$, Joseph A. Tweed ${ }^{1 *}$, Ravi Visswanathan ${ }^{2}$, James P. Saunders ${ }^{1}$, Zhenhua $\mathrm{Gu}^{3}$ and Christopher 5 L. Holliman ${ }^{1}$

$6 \quad{ }^{1}$ Medicine Design, Pfizer Worldwide Research and Development, Pfizer Inc., Groton, CT 06340

$7 \quad{ }^{2}$ Medicine Design, Pfizer Worldwide Research and Development, Pfizer Inc., San Diego, CA 92121

${ }_{8}^{3}$ Regeneron Pharmaceuticals, Inc., Tarrytown, New York 105910

10 * To whom correspondence should be addressed:

11 Pfizer, Inc., Eastern Point Road, MS8118D-2047, Groton, CT 06340

12 E-mail: joseph.tweed@pfizer.com Fax: 860.686.0775 
Table of Contents:

15 Released Nanoparticle Assay Method Development

16 Automation of the Released Nanoparticle Assay

17 Figure S1: Elution profile of PF-05212384 (500 ng/mL) on HLB sorbent

18 Figure S2: Elution profile of PF-05212384 (500 ng/mL) on cation exchange sorbent

19 Figure S3: Nanoparticle drug $(250 \mu \mathrm{g} / \mathrm{mL})$ displacement

20 $\quad$ Figure S4: Representative LC-MS/MS chromatograms of total nanoparticle monkey plasma extracts

${ }_{21} \quad$ Figure S5: Representative LC-MS/MS chromatograms of released nanoparticle monkey plasma extracts

${ }_{22}$ Table S1: Intra and Inter-day precision $(\% \mathrm{CV})$ and accuracy $(\% \mathrm{RE})$ results from the TOTAL nanoparti-

23. cle assay validation

${ }_{24}$ Table S2: Intra and Inter-day precision (\% CV) and accuracy (\%RE) results from the RELEASED nano-

${ }_{25} \quad$ particle assay validation

${ }_{26} \quad$ Table S3: Precision (\%CV) and accuracy (\%RE) stability results from the TOTAL nanoparticle assay

27 validation 


\section{Method Development (Released Assay)}

Based upon our experience with PF-05212384 (released nanoparticle drug) in our discovery bioanalysis SPE assay, we observed low process efficiency (absolute recovery). The GLP assay, however, required a thorough analysis of this observed low recovery and a SPE titration experiment was performed on PF-05212384 with the goal of striking a balance between finding a suitable extraction efficiency (relative recovery) of the small molecule drug PF-05212384 while thoroughly displacing all encapsulated drug (PF-07034663) from the stationary phase. Solid phase extraction plates with different sorbent chemistries and from various vendors were evaluated during the released NP assay development. From those preliminary experiments, two Waters Oasis micro elution (uElution) plates were chosen due to their consistent and reproducible extraction efficiency and ease of use in our assay workflow. The first uElution plate was the Waters HLB sorbent characterized by a hydrophilic-lipophilic balanced co-polymer and the second uElution plate was a MCX mixed-mode cation exchange sorbent containing a modified HLB sorbent with an ion exchange functional group addition of SO3-. The comparative work detailing the two sorbent chemistries related to optimal sample fortification and extraction conditions for the released nanoparticle assay is discussed below. A fixed plasma volume and concentration of PF-05212384 and its stable label internal standard (SLIS) was loaded onto the respective SPE sorbent beds and subsequently collected and analyzed via LC-MS/MS. To evaluate optimal separation of the released PF-05212384 from the encapsulated drug, data of two sorbents, namely HLB and MCX, were compared. The HLB stationary phase was conditioned with 2 column volumes of methanol followed by water and a final conditioning with plasma. PF-05212384 was loaded onto the HLB sorbent in diluted plasma (plasma was diluted with DPBS, $\mathrm{pH}$ 7.4). The elution profile was expressed by plotting the peak area versus solution composition (i.e. from $100 \%$ water to $100 \%$ acidified organic with $10 \%$ increasing interval). The titration profile of PF-05212384 on the HLB sorbent was obtained in three discrete elutions and is detailed in the Supporting Information. The titration experiment demonstrated that a moderate $(>20 \%)$ organic composition resulted in the elution of PF-05212384 from the stationary phase with the majority of which was eluted in a single elution step. Since PF-05212384 has a pKa of 
$\sim 8-9$, at $\mathrm{pH} 7.4$, when loaded onto the cartridge, will exist partially ionized, and as a result, less than complete retention on the cartridge was observed. PF-05212384 is retained on the cartridge when the elution solvent is $100 \%$ water and exhibits a steep elution from the stationary phase at $>20 \%$ organic (Supporting Information, Figure S1).

To selectively elute all the encapsulated drug from the cartridge, we required $~ 10 \%$ organic in water, which also resulted in some co-elution of released PF-05212384 from the cartridge, impacting the overall extraction efficiency of PF-05212384. This was of particular concern during assay development knowing that an extensive washing protocol would be needed to ensure the encapsulated drug was sufficiently removed during the SPE as to not impact the determined concentrations of the "released" PF05212384 from "artifact" drug derived from disrupted nanoparticle. Our findings demonstrated the observed poorer assay sensitivity correlated with the low extraction efficiency of PF-05212384 during the SPE procedure and is in agreement with the low extraction recovery ( $20-30 \%)$ observed using the HLB sorbent bed in the discovery assay. One important reason for this low extraction recovery is that the $\mathrm{pH}$ conditions of the sample fortification procedure and SPE conditioning steps are purposefully held at neutral $\mathrm{pH}(\sim 6.5-7.4)$ to help maintain the integrity of the nanoparticle, however resulting in a portion of the PF-05212384 exhibiting partial ionization. Because of the less than optimal results, the HLB sorbent was not a preferred choice for the GLP assay.

In contrast, in high water/low organic conditions, PF-05212384 was well retained by cation exchange sorbent MCX as detailed in the Supporting Information, Figure S2. MCX was selected as the SPE sorbent to be used for the GLP assay. The choice of MCX was demonstrated by exploiting the physicochemical properties of PF-05212384, i.e. basic molecule (pKa 8-9, ionized at acidic $\mathrm{pH}$ and ion-pair interaction with cation exchange sorbent. Quantitative elution from the cation exchange sorbent is achieved at basic $\mathrm{pH}$ since PF-05212384 becomes non-ionized). Cold water was selected as the wash solution used to displace the encapsulated nanoparticle drug from the MCX sorbent because it was costeffective and easy to volumetrically manipulate using our automation hardware. This approach was taken because any small amount of encapsulated residue (artifact) that remained on the SPE sorbent bed 
non-selectively could be disrupted in either the organic washes or elution steps of the SPE protocol. As a result of this disruption, the concentration of the released PF-05212384 drug in circulating plasma samples would be quantitated inaccurately with a high bias. Therefore, it was critical to know how many washing steps were sufficient to completely displace the nanoparticle drug from the MCX sorbent prior to eluting the released PF-05212384.

To do this, a fixed volume and concentration of encapsulated nanoparticle drug in monkey plasma $(\sim 250 \mu \mathrm{g} / \mathrm{mL})$ was loaded onto preconditioned MCX SPE sorbent plates (preconditioned with methanol, water, and DPBS buffer), and each was washed with either cold water or blank plasma by centrifugation. In order to get a general assessment of how much encapsulated drug was being displaced, solutions collected in each washing step were analyzed by taking a portion, adding acetonitrile for protein precipitation, and diluting with water. The volume normalized peak area of each washing step was compared with the peak area of the initial sample, which was calculated as the percentage of displaced (\%). For comparison, parallel experiments were conducted on HLB sorbent plates using cold water and blank plasma wash separately. It was determined that approximately $90 \%$ of the encapsulated nanoparticle drug was displaced from both MCX and HLB sorbent beds after two steps of washing by either cold water or blank plasma, and approximately $84 \%$ of the encapsulated nanoparticle drug was non-retained from the SPE plates after initial sample loading. This result supported the separation mechanism that the polymeric nanoparticle bound non-selectively to MCX and HLB SPE sorbent beds. The remaining encapsulated drug (approximately 10\%) on the SPE sorbent was collected and analyzed. Although there was a viable wash protocol to remove encapsulated nanoparticle drug with both SPE sorbents, HLB would not sufficiently bind the released PF-05212384 selectively in water (likely the water $\mathrm{pH}<7$ due to dissolved $\mathrm{CO} 2$ resulted in partially ionized PF-05212384 and as a result, binding to the polymeric stationary phase would be reduced substantially). By contrast, we had better retention of the PF-05212384 on the MCX sorbent during washing off the encapsulated nanoparticle drug by water. As a result, further washing experiments were conducted on MCX sorbent and the results are detailed in the Supporting Information, Figure S3. 
Encapsulated nanoparticle drug was displaced gradually from the MCX sorbent when washed with $100 \mu \mathrm{L}$ of cold water in each step, resulting in $99.4 \%$ being removed in 6 steps and $99.9 \%$ being removed within 12 steps. It was later demonstrated that encapsulated nanoparticles of PF-05212384 at $250 \mu \mathrm{g} / \mathrm{mL}$ and $500 \mu \mathrm{g} / \mathrm{mL}$ could be completely removed from the MCX sorbent bed within 6 washes by increasing each wash volume of cold water to $300 \mu \mathrm{L}$ per step. As a result, $300 \mu \mathrm{L}$ of cold water in each of the 6 washing steps was selected for our continuous method development and validation to ensure complete displacement of the encapsulated nanoparticle drug from the sorbent and for the accurate quantitation of released PF-05212384 concentration. After completely displacing the encapsulated nanoparticles which were a major interference to the quantitation of the released PF-05212384 concentration, biological matrices, e.g. phosphate lipids, became the next interference to remove in order to avoid a significant impact on LC-MS/MS analysis. The results from the titration experiments showed that PF05212384 tended to have a binding affinity to the MCX sorbent when using an acidified methanol solution consistent with the anticipated ion pair interaction between PF-05212384 and cation ion exchange resin. Based on this data, an acidified methanol solution was found to be a suitable washing solution to displace residual biological material following the displacement of the encapsulated drug. In addition to the experimental findings, it is important to note that our complete understanding of the compound's physiochemical properties (i.e. $\sim \mathrm{pKa}$ ) aided in optimization of all aspects of the sample preparation and extraction procedure. Therefore, two subsequent matrix eliminating wash steps were applied to the MCX sorbent beds using methanol: water: formic acid, 5:95:2 (v:v:v) followed by methanol: water: formic acid, 40:60:2 (v:v:v) prior to final elution. Interference to the quantitation of released PF-05212384 from both encapsulated nanoparticle drug and biological matrices was removed before final elution and analysis.

\section{Automation (Released Assay)}

In order to provide greater control of the released nanoparticle sample preparation and extraction procedure, the automation hardware was customized with temperature controlled labware (maintained at $4^{\circ} \mathrm{C}$ ) and a new GUI specifically designed for complex SPE workflows that require non-standard 
loading, washing and elution protocols. Since all plasma sample aliquotting was performed at $4^{\circ} \mathrm{C}$, all liquid class volumes were optimized to ensure that any differences observed in pipetting accuracy at $4^{\circ} \mathrm{C}$ were accounted for when using the customized temperature controlled labware. Due to the discrete concentration difference between encapsulated nanoparticle and the released drug in the plasma sample, fixed height dispensing was designed for extraction solvent transfer to SPE wells to avoid potential cross contamination. Prior to extraction, each extraction solution was prefilled into individual wells of a 2-mL 96-well plate to minimize cross contamination, and therefore to allow repeated use of the same set of pipetting tips during multiple steps of SPE washing or elution. All the liquid handling in the sample extraction and processing steps were performed by a 96 CO-RE pipetting head on a Hamilton Microlab STAR. $300 \mu \mathrm{L}$ pipetting tips were employed in all robot liquid transferring except for the elution steps which used $50 \mu \mathrm{L}$ pipetting tips. The liquid handling workstation was maintained at $4^{\circ} \mathrm{C}$ for all the samples, processing plates, and solutions during the entire sample handling and extraction procedure.

Critical to the overall SPE assay reproducibility and quality is the loading step of the automated SPE protocol. The dispense height of the 96 multi-channel pipetting head must be properly adjusted prior to loading the fortified biological matrix sample onto the SPE plate. Through surface tension, no droplets, residual spray or aerosol result in the minimization of any potential cross-contamination during the extraction process. Additionally, the use of an automated on-deck negative pressure vacuum manifold increased the speed and efficiency of the SPE processing. The automated SPE method also allowed the user additional flexibility to pause after each SPE extraction step to apply additional negative pressure or off-line centrifugation if needed. 


\section{Waters HLB Elution 1-3 Analyte}

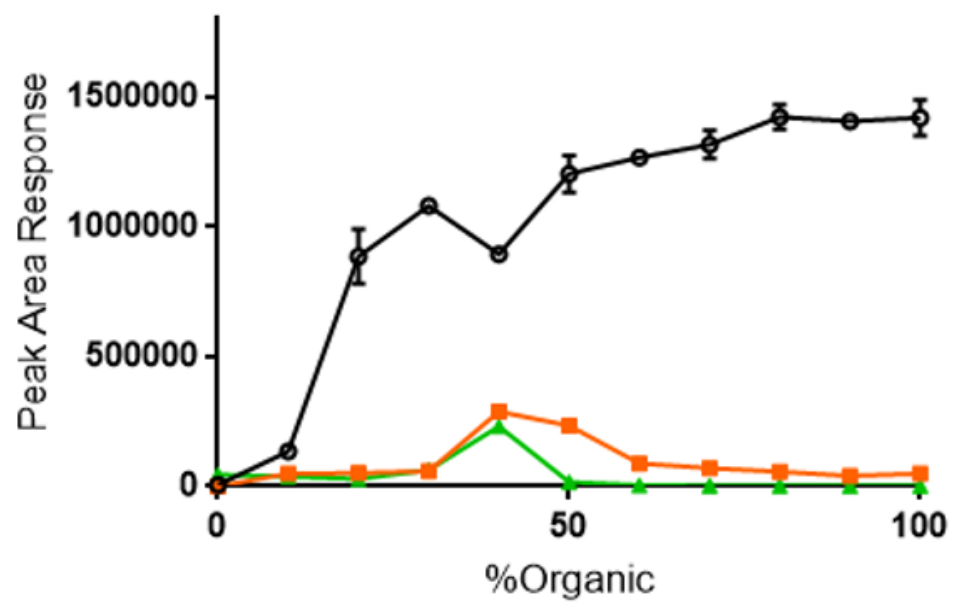

\section{$\rightarrow$ Analyte Elution 1 \\ - - Analyte Elution 2 \\ $\rightarrow \quad$ Analyte Elution 3}

Figure S1. Elution profile of PF-05212384 (500 ng/mL) on HLB sorbent when titration with organic from 0 (water) to $100 \%$ with $10 \%$ intervals. Data are expressed as mean peak area response \pm SD $(n=3)$. Tested organic solutions are methanol: acetonitrile (1:1) with $0.5 \%$ formic acid. Each sample was eluted and collected in three steps by specific organic composition for LC-MS/MS analysis. 
Figure S2. Elution profile of PF-05212384 (500 ng/mL) on cation exchange sorbent MCX when titrating with organic solution from 0 (water) to $100 \%$ with $10 \%$ interval of increase. Tested organic solutions are methanol, methanol with $2 \%$ ammonium hydroxide, and methanol with $2 \%$ formic acid. Each sample was eluted and collected for LC-MS/MS analysis. 


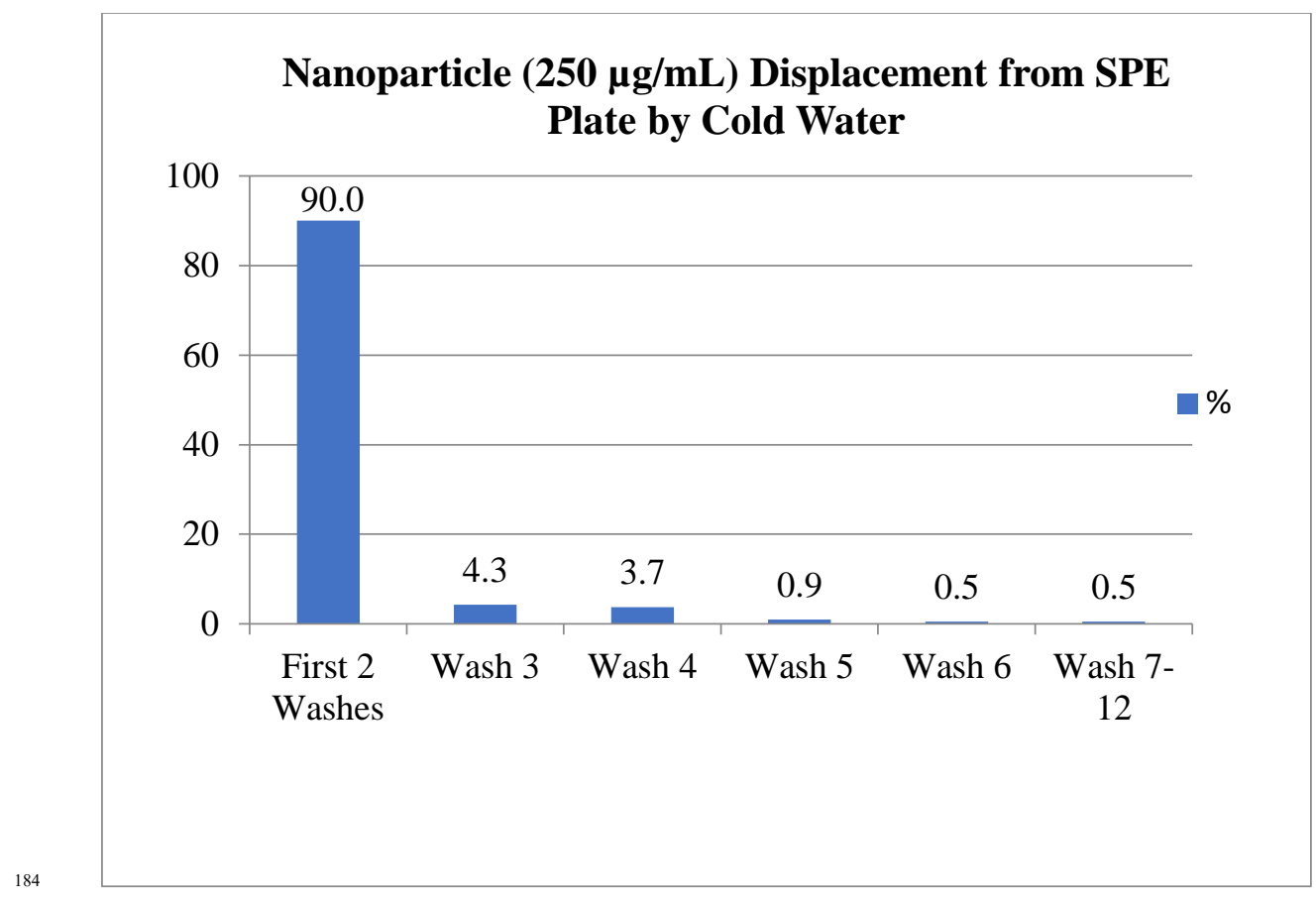

Figure S3. Nanoparticle drug $(250 \mu \mathrm{g} / \mathrm{mL})$ displacement distribution (\%) in each major washing step off a MCX SPE sorbent by water and force of centrifugation. 
A

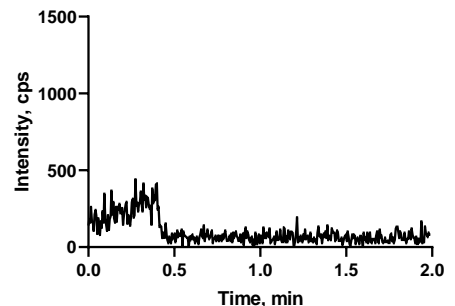

B

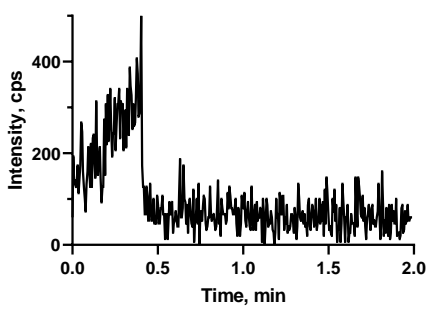

C

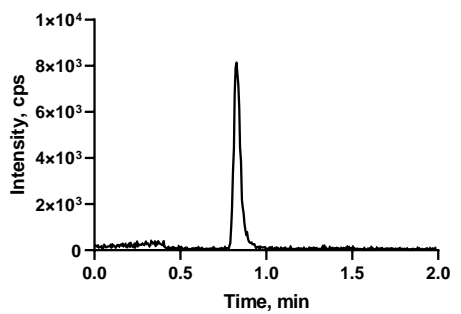

D

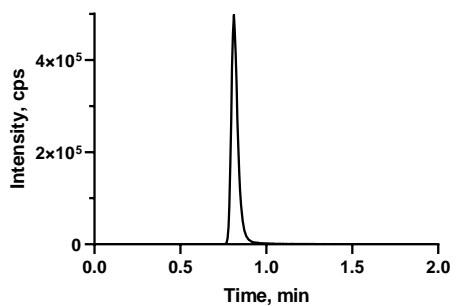

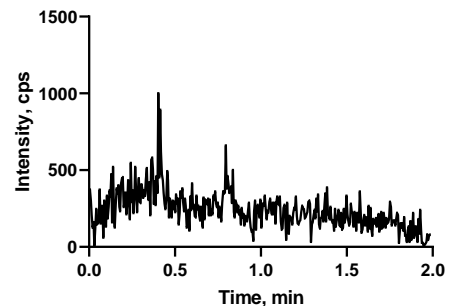
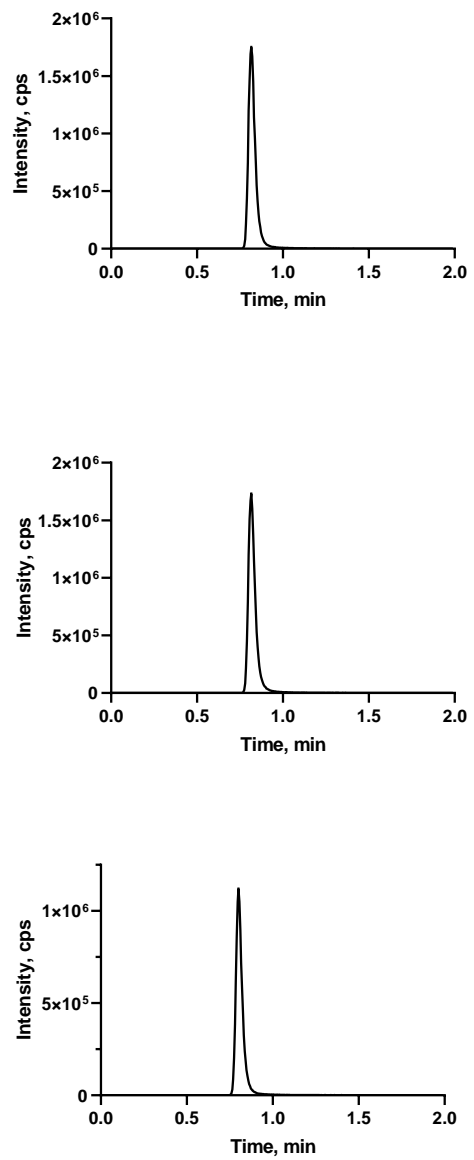

188

Figure S4: Representative LC-MS/MS chromatograms of total nanoparticle monkey plasma extracts obtained by multiple reaction monitoring (MRM) for PF-05212384 at m/z 308.9 $\rightarrow 90.0$ and its stable label internal standard at $\mathrm{m} / \mathrm{z} 312.8 \rightarrow 246.4 \mathrm{using}$ electrospray ionization (ESI). (A) double blank plasma monitored at analyte and IS, respectively, (B) blank plasma (fortified with IS) monitored at analyte and IS, respectively, (C) low limit of quantitation (LLOQ) at $10 \mathrm{ng} / \mathrm{mL}$ fortified with internal standard monitored at both channels, (D) monkey plasma biospecimen sample post IV administration of nanoparticle drug. 
A

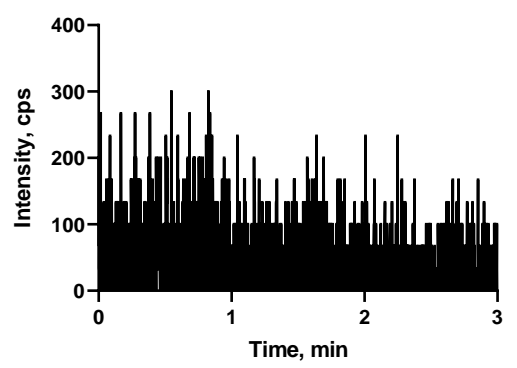

B

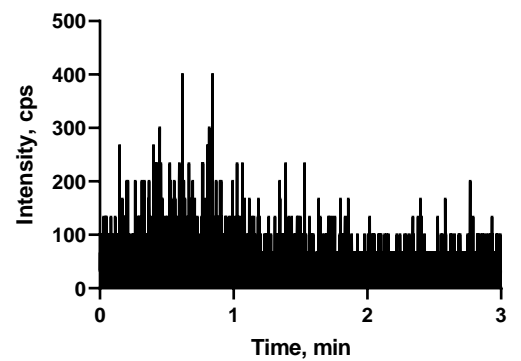

C

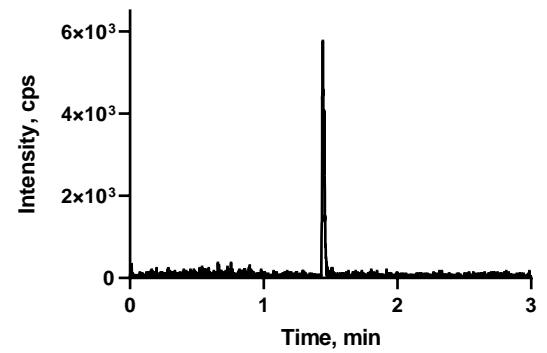

D

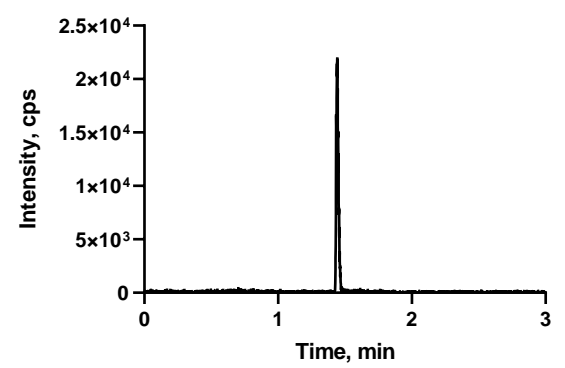

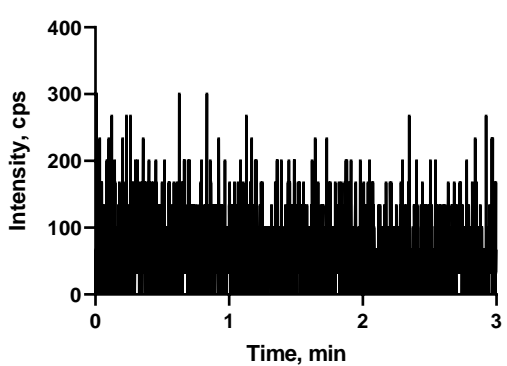
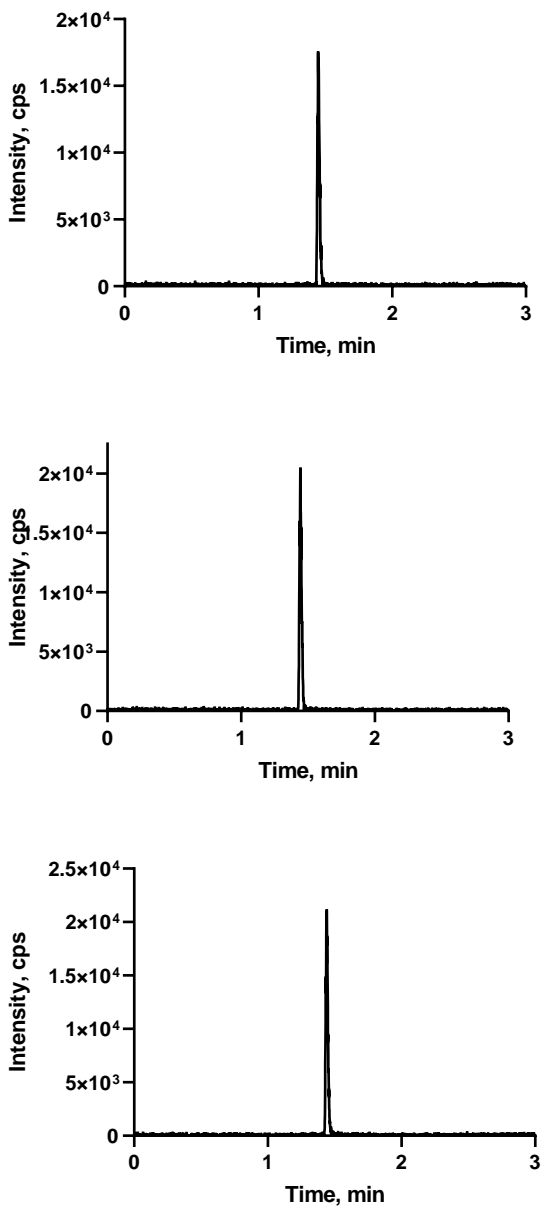

Figure S5: Representative LC-MS/MS chromatograms of released nanoparticle monkey plasma extracts obtained by multiple reaction monitoring (MRM) for PF-05212384 at m/z 308.9 $\rightarrow 90.0$ and its stable label internal standard at $\mathrm{m} / \mathrm{z} 312.8 \rightarrow 246.4$ using electrospray ionization (ESI). (A) double blank plasma monitored at analyte and IS, respectively, (B) blank plasma (fortified with IS) monitored at analyte and IS, respectively, (C) low limit of quantitation (LLOQ) at $10 \mathrm{ng} / \mathrm{mL}$ fortified with internal standard monitored at both channels, (D) monkey plasma biospecimen sample post IV administration of nanoparticle drug. 


\begin{tabular}{|c|c|c|c|c|c|c|c|c|}
\hline \multirow{3}{*}{$\begin{array}{l}\text { Nominal } \\
\text { Concentration } \\
(\mathrm{ng} / \mathrm{mL})\end{array}$} & \multicolumn{6}{|c|}{ Intra-day } & \multirow{3}{*}{$\begin{array}{c}\text { Inter-day } \\
\text { Precision }(\mathrm{n}=3) \\
(\%)\end{array}$} & \multirow{3}{*}{$\begin{array}{c}\text { Inter-day } \\
\text { Accuracy }(\mathrm{n}=3) \\
(\%)\end{array}$} \\
\hline & \multicolumn{2}{|c|}{ Validation Day \#1* } & \multicolumn{2}{|c|}{ Validation Day \#2 } & \multicolumn{2}{|c|}{ Validation Day \#3 } & & \\
\hline & $\begin{array}{c}\text { Precision } \\
(\%)\end{array}$ & $\begin{array}{c}\text { Accuracy } \\
(\%)\end{array}$ & $\begin{array}{c}\text { Precision } \\
(\%)\end{array}$ & $\begin{array}{c}\text { Accuracy } \\
(\%)\end{array}$ & $\begin{array}{c}\text { Precision } \\
(\%)\end{array}$ & $\begin{array}{c}\text { Accuracy } \\
(\%)\end{array}$ & & \\
\hline 500 & 6.7 & -12.2 & 2.8 & 1.4 & 2.9 & 7.4 & 9.1 & -0.6 \\
\hline 1500 & 8.3 & -6.0 & 2.5 & -8.0 & 2.3 & -6.0 & 5.0 & -6.7 \\
\hline 20,000 & 2.6 & 5.0 & 1.0 & 1.5 & 1.1 & 1.0 & 2.4 & 2.5 \\
\hline 200,000 & 3.6 & 0.0 & 1.7 & 3.0 & 1.9 & 3.0 & 2.8 & 2.0 \\
\hline
\end{tabular}

Intra-day, $\mathrm{N}=6$; Inter-day, $\mathrm{N}=18$

Precision: Percent coefficient of variation $(\% \mathrm{CV})$ of calculated concentration

Accuracy: Percent relative error (\%RE); [1-(experimentally determined concentration $/$ nominal concentration)] x $100 \%$

* The LIOQ QC (500 ng/mL) for Validation Day \#1 used $\mathrm{N}=5$ for intra-day statistics and $\mathrm{N}=17$ for inter-day statistics; data set contained a statistical outlier as determined by Dixon Q test at the $99 \%$ confidence interval

Table S1. Intra and Inter-day precision (\% CV) and accuracy (\%RE) results from the TOTAL nanoparticle validation of PF 07034663 (encapsulated PF 05212384) in monkey plasma using an automated protein precipitation sample preparation method.

\begin{tabular}{|c|c|c|c|c|c|c|c|c|}
\hline \multirow{3}{*}{$\begin{array}{c}\text { Nominal } \\
\text { Concentration } \\
(\mathrm{ng} / \mathrm{mL})\end{array}$} & \multicolumn{6}{|c|}{ Intra-day } & \multirow{3}{*}{$\begin{array}{c}\text { Inter-day } \\
\text { Precision }(\mathrm{n}=3) \\
(\%)\end{array}$} & \multirow{3}{*}{$\begin{array}{c}\text { Inter-day } \\
\text { Accuracy }(\mathrm{n}=3) \\
(\%)\end{array}$} \\
\hline & \multicolumn{2}{|c|}{ Validation Day \#1 } & \multicolumn{2}{|c|}{ Validation Day $\# 2 *$} & \multicolumn{2}{|c|}{ Validation Day \#3 } & & \\
\hline & $\begin{array}{c}\text { Precision } \\
(\%)\end{array}$ & $\begin{array}{c}\text { Accuracy } \\
(\%)\end{array}$ & $\begin{array}{c}\text { Precision } \\
(\%)\end{array}$ & $\begin{array}{c}\text { Accuracy } \\
(\%)\end{array}$ & $\begin{array}{c}\text { Precision } \\
(\%)\end{array}$ & $\begin{array}{c}\text { Accuracy } \\
(\%)\end{array}$ & & \\
\hline 10.0 & 4.9 & 7.0 & 6.6 & 5.0 & 4.1 & -2.1 & 6.3 & 3.0 \\
\hline 30.0 & 5.4 & 0.3 & 2.6 & 1.7 & 1.6 & -2.7 & 3.8 & -0.3 \\
\hline 150 & 6.6 & 1.3 & 8.8 & 11.3 & 1.7 & 4.7 & 7.2 & 5.3 \\
\hline 750 & 5.7 & 11.5 & 10.3 & 3.9 & 4.6 & 10.1 & 7.4 & 8.5 \\
\hline
\end{tabular}

Intra-day, $\mathrm{N}=6$; Inter-day, $\mathrm{N}=18$

Precision: Percent coefficient of variation $(\% \mathrm{CV})$ of calculated concentration

Accuracy: Percent relative error (\%RE); [1-(experimentally determined concentration /nominal concentration)] x $100 \%$

* The mid QC $(150 \mathrm{ng} / \mathrm{mL})$ for Validation Day $\# 2$ used $\mathrm{N}=5$ for intra-day statistics and $\mathrm{N}=17$ for inter-day statistics; data set contained a laboratory processing error associated with an assignable cause

Table S2. Intra and Inter-day precision (\%CV) and accuracy (\%RE) results from the RELEASED nanoparticle validation of PF 05212384 in monkey plasma using automated solid phase extraction (SPE, MCX) method.

\begin{tabular}{|c|c|c|c|c|c|c|c|}
\hline \multirow{3}{*}{$\begin{array}{c}\text { Quality Control } \\
\text { Sample } \\
\text { PF-07034663 }\end{array}$} & \multirow{3}{*}{$\begin{array}{l}\text { Nominal Conc } \\
(\mathrm{ng} / \mathrm{mL})\end{array}$} & \multicolumn{6}{|c|}{ Storage Stability } \\
\hline & & \multicolumn{2}{|c|}{69 Days at $-20^{\circ} \mathrm{C}$} & \multicolumn{2}{|c|}{$\begin{array}{c}\text { Freeze/ Thaw } \\
\text { (6 Cycles) }\end{array}$} & \multicolumn{2}{|c|}{$\begin{array}{l}16 \text { Hours } \\
\text { at Ambient }\end{array}$} \\
\hline & & $\begin{array}{c}\text { Mean Conc }(\mathrm{ng} / \mathrm{mL}) \\
\text { with }(\mathrm{CV} \%)(\mathrm{N}=6)\end{array}$ & $\begin{array}{c}\text { Accuracy } \\
(\%)\end{array}$ & $\begin{array}{c}\text { Mean Conc (ng/mL) } \\
\text { with }(\mathrm{CV} \%)(\mathrm{N}=6)\end{array}$ & $\begin{array}{c}\text { Accuracy } \\
(\%)\end{array}$ & $\begin{array}{c}\text { Mean } \operatorname{Conc}(\mathrm{ng} / \mathrm{mL}) \\
\text { with }(\mathrm{CV} \%)(\mathrm{N}=6)\end{array}$ & $\begin{array}{c}\text { Accuracy } \\
(\%)\end{array}$ \\
\hline LQC & 1,500 & $1,530(2.3 \%)$ & 2.0 & $1,550(7.0 \%)$ & 3.3 & $1,580(2.0 \%)$ & 5.3 \\
\hline $\mathrm{HOC}$ & 200,000 & $188,000(2.3 \%)$ & -6.0 & $205,000(2.2 \%)$ & 2.5 & $216,000(1.5 \%)$ & 8.0 \\
\hline
\end{tabular}

Intra-day, $\mathrm{N}=6$; Inter-day, $\mathrm{N}=18$

Precision: Percent coefficient of variation $(\% \mathrm{CV})$ of calculated concentration

Accuracy: Percent relative error ( $\% \mathrm{RE})$; [1-(experimentally determined concentration /nominal concentration)] x 100\%

Table S3. Precision (\%CV) and accuracy (\%RE) stability results from the TOTAL nanoparticle validation of PF 07034663 (encapsulated PF 05212384 ) under various storage and processing conditions including $-20^{\circ} \mathrm{C}$, freeze/thaw, $4^{\circ} \mathrm{C}$, and ambient temperature in monkey plasma. Concentration of PF-05212384 in monkey plasma is quantitated after processing validation samples using an automated protein precipitation sample preparation method. The \%RE is the relative concentration of PF 05212384 in stability samples compared to its nominal concentration. 